\title{
Comparison of Coronary Artery Calcium Scores Between Patients With Psoriasis and Type 2 Diabetes
}

Bobbak Mansouri, MD; Dario Kivelevitch, MD; Balaji Natarajan, MD; Aditya A. Joshi, MD; Caitriona Ryan, MD; Katie Benjegerdes, BS; Jeffrey M. Schussler, MD; Daniel J. Rader, MD; Muredach P. Reilly, MBBCH, MSCE; Alan Menter, MD; Nehal N. Mehta, MD, MSCE

IMPORTANCE Psoriasis is associated with an increased risk of cardiovascular diseases. Subclinical atherosclerosis in patients with psoriasis has not been compared with other conditions associated with increased cardiovascular risk and more rigorous cardiovascular disease screening, such as type 2 diabetes.

OBJECTIVE To assess the burden of asymptomatic coronary atherosclerosis measured by coronary artery calcium score in patients with moderate to severe psoriasis compared with patients with type 2 diabetes and healthy controls.

DESIGN, SETTING, AND PARTICIPANTS Three single-center, cross-sectional studies were performed in patients recruited from specialty outpatient clinics with moderate to severe psoriasis without type 2 diabetes (recruited from November 1, 2013, through April 31, 2015), patients with type 2 diabetes without psoriasis or other inflammatory diseases (recruited from July 1, 2009, through June 20, 2011), and age- and sex-matched healthy controls without psoriasis, type 2 diabetes, or other inflammatory diseases (recruited from July 1 , 2009, through June 20, 2011).

EXPOSURES Psoriasis, type 2 diabetes, and healthy control effect on coronary artery calcium score.

MAIN OUTCOMES AND MEASURES Coronary artery calcium measured by Agatston score.

RESULTS A total of 387 individuals participated in the study. Mean (SD) age was 51 (7.7), 52 (8.0), and 52 (8.0) years in the psoriasis, type 2 diabetes, and healthy control cohorts, respectively. There were 64 men (49.6\%) in each group, and most patients were white (119 [92.2\%], 123 [95.3\%], and 128 [99.2\%] in the psoriasis, type 2 diabetes, and healthy control cohorts, respectively). Patients with psoriasis had low cardiovascular risk measured by the Framingham Risk Score but had a high prevalence of cardiovascular and cardiometabolic risk factors, similar to patients with type 2 diabetes. In a fully adjusted model, psoriasis was associated with coronary artery calcium (Tobit regression ratio, 0.89; $P<.001$ ) similar to the association in type 2 diabetes (Tobit regression ratio, $0.79 ; P=.04$ ). Likelihood ratio testing revealed incremental value for psoriasis in a fully adjusted model $\left(\chi^{2}=4.48, P=.03\right)$ in predicting coronary artery calcium. Psoriasis was independently associated with the presence of any coronary artery calcium (odds ratio, $2.35 ; 95 \% \mathrm{Cl}, 1.12-4.94$ ) in fully adjusted models, whereas the association of coronary artery calcium with type 2 diabetes was no longer significant after adding body mass index to the model (odds ratio, $2.18 ; 95 \% \mathrm{Cl}, 0.75-6.35$ ).

CONCLUSIONS AND RELEVANCE Patients with psoriasis have increased coronary artery calcium by mean total Agatston scores, similar to that of patients with type 2 diabetes, suggesting that patients with psoriasis harbor high rates of subclinical atherosclerosis beyond adjustment for body mass index. Major educational efforts for patients and physicians should be undertaken to reduce the burden of cardiovascular disease in patients with psoriasis.

JAMA Dermatol. 2016;152(11):1244-1253. doi:10.1001/jamadermatol.2016.2907 Published online August 24, 2016.
Supplemental content
Author Affiliations: Author affiliations are listed at the end of this article.

Corresponding Author: Nehal N. Mehta, MD, MSCE, Section of Inflammation and Cardiometabolic Diseases, National Heart, Lung, and Blood Institute, National Institutes of Health, 10 Center Dr, Bethesda, MD 20814 (nehal.mehta@nih.gov). 
$\mathrm{P}$ soriasis is an immune-mediated genetic disease associated with systemic inflammation that affects approximately $2 \%$ of North American and European populations. Prevalence varies based on racial and geographic differences. ${ }^{1}$ Approximately $20 \%$ to $25 \%$ of patients have moderate to severe disease, requiring systemic therapy. ${ }^{2}$ Up to onethird of patients with psoriasis develop psoriatifc arthritis. Psoriasis is strongly linked with several behavioral and medical comorbidities, including the metabolic syndrome ${ }^{3}$ and cardiovascular disease. ${ }^{4}$

Psoriasis appears to be an independent risk factor for coronary artery disease, myocardial infarction, cerebrovascular disease, stroke, peripheral vascular disease, and cardiovascular mortality. ${ }^{5-8}$ Patients with psoriasis have an excess risk of cardiovascular mortality, which is the leading cause of mortality in this group, a 4- to 5-year younger mean age at death compared with those without psoriasis, ${ }^{9}$ and an increased major adverse cardiovascular events risk, particularly those who have severe psoriatic disease or have not been prescribed diseasemodifying antirheumatic drugs. ${ }^{10}$ Furthermore, the presence of severe psoriasis confers an additional $6.2 \%$ absolute excess risk of 10-year major adverse cardiovascular events, even after adjusting for age, sex, type 2 diabetes, hypertension, tobacco use, and hyperlipidemia. ${ }^{11}$ Framingham Risk Scores (FRSs) underestimated cardiovascular risk in $73 \%$ of low-risk and $53 \%$ of high-risk patients with psoriasis, who were reclassified to higher-risk categories. ${ }^{12}$ Despite these substantial data, patients with psoriasis are not actively educated, counseled, or screened for cardiovascular disease because data have been limited by the retrospective nature of population-based studies $^{5-10,13}$ and the limited power of prospective studies ${ }^{14,15}$ using novel imaging methods.

Surrogate outcome measures quantifying cardiovascular risk are important. Coronary artery calcium (CAC) assessment has become widely accepted as a true measure of the total burden of atherosclerosis and the cornerstone for screening the risk of future cardiac events and improving cardiovascular risk stratification beyond traditional risk factors, especially in higher-risk groups. ${ }^{16,17}$ Three studies ${ }^{18-20}$ found an association between moderate to severe psoriasis and increased CAC, yet cardiovascular risk stratification for patients with psoriasis remains the same as the general population despite increasing evidence to support this important excess in cardiovascular risk.

Type 2 diabetes mellitus provides an important model of an established, high-risk disease associated with increased cardiovascular risk for comparison. Patients with psoriasis have atherogenic lipoprotein ${ }^{21}$ and adipokine ${ }^{22}$ profiles similar to those in patients with type 2 diabetes. Furthermore, CAC assessment is superior to traditional cardiovascular risk factors for predicting silent myocardial ischemia and short-term outcomes, ${ }^{23}$ myocardial infarction and cardiac death, ${ }^{24}$ and cardiovascular events (including stroke) ${ }^{25}$ in asymptomatic type 2 diabetes. As a result, the American College of Cardiology/ American Heart Association guidelines now recommend computed tomography for CAC assessment to improve cardiovascular risk stratification in asymptomatic patients 40 years or older with type 2 diabetes. ${ }^{26}$ The objective of this study was

\section{Key Points}

Question How does the burden of asymptomatic coronary atherosclerosis measured by coronary artery calcium score in patients with moderate to severe psoriasis compare with patients with type 2 diabetes and healthy controls?

Findings In this analysis of data from 3 cross-sectional studies, the prevalence of moderate to severe coronary calcification was similar between patients with psoriasis and those with type 2 diabetes and approximately 5 times greater than healthy controls. Coronary calcium in patients with moderate to severe psoriasis was similarly associated with known cardiovascular and cardiometabolic risk factors when compared with patients with type 2 diabetes, and the presence of moderate to severe psoriasis was a significantly stronger predictor of coronary calcification than type 2 diabetes, independent of the effect of known cardiovascular and cardiometabolic risk factors.

Meaning These findings support screening for cardiovascular risk factors systematically in patients with moderate to severe psoriasis and type 2 diabetes.

to compare CAC scores in patients with psoriasis with CAC scores in patients with type 2 diabetes based on the assumption that comparable increases in CAC scores would support the need for heightened awareness and appropriate screening for cardiovascular disease in patients with psoriasis.

\section{Methods}

Our study population was composed of unrelated individuals recruited from a specialty psoriasis clinic at the Baylor University Medical Center (BUMC), the Penn Diabetes Heart Study (PDHS), ${ }^{27}$ and the Philadelphia Area Metabolic Syndrome Network (PAMSyN). ${ }^{21}$ The cohorts represent patients with moderate to severe psoriasis without type 2 diabetes (BUMC), type 2 diabetes without psoriasis or other inflammatory diseases (PDHS), and age- and sex-matched healthy controls without psoriasis, type 2 diabetes, or other inflammatory diseases (PAMSyN). Patients in all cohorts had no history of coronary heart disease. Patients with psoriasis were consecutively screened for potential study inclusion from November 1, 2013, through April 31, 2015. Patients with type 2 diabetes without psoriasis or other inflammatory diseases were recruited from July 1, 2009, through June 20, 2011, and age- and sexmatched healthy controls without psoriasis, type 2 diabetes, or other inflammatory diseases were recruited from July 1 , 2009, through June 20, 2011. Treated and untreated patients with psoriasis were included and required a diagnosis of moderate to severe psoriasis defined as having 1 of the following 4 criteria: (1) body surface area involvement greater than $10 \%$, (2) Psoriasis Area and Severity Index score greater than 10, (3) Dermatology Life Quality Index score greater than 10, or (4) treatment with oral systemic and/or biological therapy for at least 6 months. Patients with a history of cardiovascular disease or symptoms, type 2 diabetes (defined as self-reported history, current use of medications, or fasting blood glucose level $>126 \mathrm{mg} / \mathrm{dL}$ [to convert to millimoles per liter, multiply 
by 0.0555]), comorbid inflammatory disease (excluding psoriatic arthritis), or current or prior malignant tumor (excluding nonmelanoma skin cancers) were excluded. Both the PDHS and PAMSyN excluded patients with prior myocardial infarction, coronary revascularization, angiographic disease, a positive stress test result, creatinine level greater than $1.2 \mathrm{mg} / \mathrm{dL}$ (to convert to micromoles per liter, multiply by 88.4 ), and, in PAMSyN only, the presence of type 2 diabetes. The institutional review boards from Baylor University Medical Center and the University of Pennsylvania approved all 3 studies. All patients provided written informed consent before participation, and data were deidentified.

\section{Evaluated Parameters}

Metabolic syndrome was defined as the presence of more than 2 of the following: waist circumference greater than $102 \mathrm{~cm}$ (male) or greater than $89 \mathrm{~cm}$ (female), triglyceride level of 150 $\mathrm{mg} / \mathrm{dL}$ or higher (to convert to millimoles per liter, multiply by 0.0113), high-density lipoprotein cholesterol (HDL-C) level less than 40 (male) or less than 50 (female) mg/dL (to convert to millimoles per liter, multiply by 0.0259), fasting blood glucose level of $100 \mathrm{mg} / \mathrm{dL}$ or higher, or blood pressure greater than $130 / 85 \mathrm{~mm} \mathrm{Hg}$ or treated hypertension. ${ }^{28}$ The FRS was calculated using total cholesterol values. ${ }^{29}$ The CAC scores were expressed as mean total Agatston score and percentile scores for all 3 cohorts. ${ }^{30}$ More detailed methods can be found in the eMethods in the Supplement.

The 3 cohorts (BUMC, PDHS, and PAMSyN) were age and sex matched. Summaries of continuous variables are reported as mean (SD) for normally distributed data and as median (interquartile range) for nonnormally distributed data and categorical variables as proportions and percentages. Simple unpaired, 2-tailed $t$ tests (for parametric data) and MannWhitney tests (for nonparametric data) were performed for continuous variables and $\chi^{2}$ tests for categorical variables, respectively. Univariate analysis of CAC, assessed by mean total Agatston scores, was performed using Tobit censored regression of natural $\log (\mathrm{CAC}+1)$ because of the markedly skewed distribution of CAC in all 3 cohorts. ${ }^{31}$ Crude associations between CAC and study variables were also explored using a Spearman correlation analysis (eTable 1 in the Supplement). Multivariable Tobit hierarchical regression analysis was performed to understand the association between the presence of disease (psoriasis or type 2 diabetes) and CAC after adjusting for groups of confounding variables. Model 1 adjusted for age and sex, model 2 additionally adjusted for established cardiovascular risk factors (low-density lipoprotein cholesterol [LDL-C] level, HDL-C level, triglyceride level, systolic blood pressure, fasting blood glucose level, and current tobacco use), model 3 additionally adjusted for cardiometabolic risk factors (body mass index [BMI] and metabolic syndrome), and model 4 additionally adjusted for medication effects on coronary calcium levels (aspirin, angiotensin-converting enzyme inhibitors, and statins). Likelihood ratio testing was performed to assess the incremental effect of disease state (psoriasis and type 2 diabetes) to each model, and $\chi^{2}$ and $P$ values for each model were reported. A logistic adjusted regression analysis was also performed to account for the high percent- age of CAC scores of $\mathrm{O}$ in all groups. The CAC data were converted into categorical data (1 indicating any calcium and 0 indicating no calcium) to understand the effect of the disease (psoriasis or type 2 diabetes) on the presence of any calcium in the coronary arteries (CAC score $>0$ ), which were reported as odds ratios (ORs) with $95 \%$ CIs. All analyses were performed using STATA statistical software, version 12.0 (StataCorp), and $P \leq .05$ was set as the threshold of statistical significance.

Our sample had more than $90 \%$ power to detect an OR of 2.3 to 2.5 using the group of individuals free of psoriasis as reference, with a frequency of nonzero CAC scores of 0.45 in patients with psoriasis and 0.3 in individuals without psoriasis. Considerations of nonzero CAC score frequencies were based on previous studies. ${ }^{18,19}$

\section{Results}

\section{Characteristics of Study Groups}

Table 1 summarizes patients' demographic and clinical characteristics. A total of 387 individuals participated in the study. Mean (SD) age was 51 (7.7), 52 (8.0), and 52 (8.0) years in the psoriasis, type 2 diabetes, and healthy control cohorts, respectively. There were 64 men (49.6\%) in each group, and most patients were white (119 [92.2\%], 123 [95.3\%], and 128 [99.2\%] in the psoriasis, type 2 diabetes, and healthy control cohorts, respectively). A significantly high prevalence of cardiovascular and cardiometabolic risk factors was noted in the psoriasis and type 2 diabetes cohorts, with the type 2 diabetes cohort having the highest prevalence of hypertension (49 [38.0\%] in the psoriasis cohort vs 71 [55.0\%] in the type 2 diabetes cohort, $P=.006)$ and waist circumference (99.75 [16.8] $\mathrm{cm}$ in the psoriasis cohort vs 108.0 [15.8] cm in the type 2 diabetes cohort, $P=.001)$. Dyslipidemia was most prevalent in the psoriasis cohort (107 [82.9\%] in the psoriasis cohort vs 81 [62.8\%] in the type 2 diabetes cohort, $P<.001)$. However, the type 2 diabetes group had significantly higher triglyceride levels (median, $100 \mathrm{mg} / \mathrm{dL}$ [interquartile range, 77-138 $\mathrm{mg} / \mathrm{dL}$ ] for the psoriasis cohort vs $132 \mathrm{mg} / \mathrm{dL}$ [interquartile range, 92-174 $\mathrm{mg} / \mathrm{dL}$ ] for the type 2 diabetes cohort; $P=.002$ ) and lower HDL-C levels $(57.7$ [19.2] mg/dL for the psoriasis cohort vs 47.1 [11.4] mg/dL for the type 2 diabetes cohort, $P<.001)$. The control group had greater total cholesterol (175.6 [37.2] $\mathrm{mg} / \mathrm{dL}$ in the type 2 diabetes cohort vs 204.6 [36.6] in the control cohort, $P<.001)$ and LDL-C levels (99.1 [28.7] $\mathrm{mg} / \mathrm{dL}$ in the type 2 diabetes cohort vs 122.5 [32.2] $\mathrm{mg} / \mathrm{dL}$ in the control cohort, $P<.001)$. The psoriasis and type 2 diabetes cohorts had a similar distribution of CAC in higher tertiles (CAC score $>100$ and CAC score $>400$, respectively) compared with the healthy control cohort.

\section{Characteristics of the Psoriasis Cohort}

Table 2 summarizes the psoriasis cohort characteristics stratified by the presence of coronary calcium (CAC score of 0 and CAC score $>0)$. Patients with psoriasis $(n=129)$ had median disease duration of 11 years. The mean age of psoriasis onset was 27.8 years, with a later onset in patients with CAC scores 


\begin{tabular}{|c|c|c|c|c|c|c|}
\hline \multirow[b]{2}{*}{ Characteristic } & \multirow[b]{2}{*}{$\begin{array}{l}\text { Psoriasis } \\
\text { Cohort } \\
(n=129)\end{array}$} & \multirow{2}{*}{$\begin{array}{l}\text { Type } 2 \\
\text { Diabetes } \\
\text { Cohort } \\
(n=129)\end{array}$} & \multirow[b]{2}{*}{$\begin{array}{l}\text { Healthy } \\
\text { Controls } \\
(n=129)\end{array}$} & \multicolumn{3}{|l|}{$P$ Value $^{\mathrm{b}}$} \\
\hline & & & & $\begin{array}{l}\text { Psoriasis Cohort } \\
\text { vs Type } 2 \\
\text { Diabetes Cohort }\end{array}$ & $\begin{array}{l}\text { Psoriasis Cohort } \\
\text { vs Healthy } \\
\text { Controls }\end{array}$ & $\begin{array}{l}\text { Type } 2 \\
\text { Diabetes Cohort } \\
\text { vs Healthy Controls }\end{array}$ \\
\hline Age, mean (SD), y & $51(7.7)$ & $52(8.0)$ & $52(8.0)$ & NA & NA & NA \\
\hline Male sex & $64(49.6)$ & $64(49.6)$ & $64(49.8)$ & NA & NA & NA \\
\hline \multicolumn{7}{|l|}{ Race/ethnicity } \\
\hline White & $119(92.2)$ & $123(95.3)$ & $128(99.2)$ & \multirow{4}{*}{.61} & \multirow{4}{*}{.05} & \multirow{4}{*}{.14} \\
\hline African American & $5(3.9)$ & $2(1.6)$ & 0 & & & \\
\hline Hispanic & $4(3.1)$ & $4(3.1)$ & $1(0.8)$ & & & \\
\hline Other & $1(0.8)$ & 0 & 0 & & & \\
\hline Hypertension & $49(38.0)$ & $71(55.0)$ & $47(36.4)$ & .006 & .80 & .003 \\
\hline Dyslipidemia & 107 (82.9) & $81(62.8)$ & $50(38.8)$ & $<.001$ & $<.001$ & $<.001$ \\
\hline Metabolic syndrome & $49(38.0)$ & $96(74.4)$ & $28(21.7)$ & $<.001$ & .006 & $<.001$ \\
\hline Current smoking & $12(9.3)$ & $16(12.4)$ & $23(17.8)$ & .42 & .04 & .22 \\
\hline Regular exercise & $60(46.5)$ & $74(57.4)$ & $71(55.0)$ & .08 & .1 & .93 \\
\hline Body mass index ${ }^{c}$ & $28.6(6.3)$ & $33.1(6.4)$ & $30.0(4.5)$ & $<.001$ & .04 & $<.001$ \\
\hline Waist circumference, $\mathrm{cm}$ & $99.75(16.8)$ & $108.0(15.8)$ & $99.6(14.0)$ & .001 & .92 & $<.001$ \\
\hline \multicolumn{7}{|l|}{$\mathrm{BP}, \mathrm{mm} \mathrm{Hg}$} \\
\hline Systolic & $129.6(18.1)$ & $125.2(14.3)$ & $125.6(16.0)$ & .03 & .05 & .83 \\
\hline Diastolic & $79.83(12.2)$ & $75.02(8.9)$ & $75.67(9.2)$ & .004 & .002 & .56 \\
\hline FRS 10-y risk, median (IQR) & $9(6-12)$ & $8(5-13)$ & $5(3-8)$ & .83 & $<.001$ & $<.001$ \\
\hline \multicolumn{7}{|l|}{ Lipid and glucose profile } \\
\hline Total cholesterol, mean (SD), mg/dL & $188.3(34.7)$ & $175.6(37.2)$ & 204.6 (36.6) & .005 & $<.001$ & $<.001$ \\
\hline LDL-C, mean (SD), mg/dL & $108.2(28.7)$ & $99.09(28.7)$ & $122.5(32.2)$ & .01 & $<.001$ & $<.001$ \\
\hline HDL-C, mean (SD), mg/dL & $57.70(19.2)$ & $47.11(11.4)$ & $55.51(14.3)$ & $<.001$ & .31 & $<.001$ \\
\hline Triglycerides, median (IQR), mg/dL & $100(77-138)$ & $132(92-174)$ & $108(88-151)$ & $<.001$ & .03 & .04 \\
\hline VLDL-C, median (IQR) & $22(13-27)$ & $27(19-38)$ & $24(17-32)$ & .09 & .37 & .03 \\
\hline Glucose, median (IQR), mg/dL & $92(86-99)$ & $119(100-146)$ & $69(62-74)$ & $<.001$ & $<.001$ & $<.001$ \\
\hline CAC score, median (IQR) & $0(0-50)$ & $1(0-76)$ & $0(0-6)$ & NA & NA & NA \\
\hline \multicolumn{7}{|l|}{ CAC score } \\
\hline 0 & $75(58.1)$ & $64(49.6)$ & $89(69.0)$ & \multirow{4}{*}{.45} & \multirow{4}{*}{.003} & \multirow{4}{*}{$<.001$} \\
\hline $1-100$ & $30(23.3)$ & $38(29.5)$ & $35(27.1)$ & & & \\
\hline $101-400$ & $19(14.7)$ & $20(15.5)$ & $4(3.1)$ & & & \\
\hline$>400$ & $5(3.9)$ & $7(5.4)$ & $1(0.8)$ & & & \\
\hline
\end{tabular}

Abbreviations: BP, blood pressure; CAC, coronary artery calcium; FRS, Framingham Risk Score; HDL-C, high-density lipoprotein cholesterol; IQR, interquartile range; LDL-C, low-density lipoprotein cholesterol; NA, not applicable; VLDL-C, very low-density lipoprotein cholesterol.

SI conversion factors: To convert total cholesterol, LDL-C, and HDL-C to millimoles per liter, multiply by 0.0259 ; triglycerises to millimoles per liter, multiply by 0.0113; glucose to millimoles per liter, multiply by 0.0555 .

${ }^{\text {a }}$ Data are presented as number (percentage) of patients unless otherwise indicated.

${ }^{\mathrm{b}} P$ values were derived from a simple unpaired, 2-tailed $t$ test for parametric variables and from the Mann-Whitney test for nonparametric variables. The $\chi^{2}$ test was used for categorical variables. $P<.05$ is considered statistically significant.

${ }^{\mathrm{C}}$ Calculated as weight in kilograms divided by height in meters squared.

greater than 0 ( 25 vs 31 years, $P=.02$ ). A total of 120 patients (93.0\%) had plaque-type psoriasis, and a significantly higher percentage of patients with plaque-type psoriasis had no evidence of CAC (74 [98.7\%] vs 46 [85.2\%] in the CAC score of 0 vs CAC score $>1$ groups, $P=.003)$. A total of 66 patients (51.2\%) had nail involvement, and 26 (20.2\%) had nonpustular involvement of their palms and/or soles. A total of 96 patients $(74.4 \%)$ were undergoing systemic therapy for a median of 3 years, with 96 patients (74.4\%) receiving biological therapy. Psoriasis disease activity was generally well controlled (median Psoriasis Area and Severity Index score, 1.2; median body surface area, $4 \%$ ), with 100 patients (77.5\%) either clear (score of 0) or having minimal (score of 1) disease based on the Physician Global Assessment. Forty-four patients (34.1\%) had concomitant psoriatic arthritis. No significant differences were observed in clinical severity scores or treatment status between the 2 groups.

\section{Association of Psoriasis and Traditional}

Cardiovascular Risk Factors With CAC

We performed a stratified analysis based on prevalence of traditional cardiovascular risk factors in patients with psoriasis (Figure). Presence of any traditional risk factor in addition to psoriasis was associated with increased CAC compared with 


\begin{tabular}{|c|c|c|c|c|}
\hline Characteristic & $\begin{array}{l}\text { All } \\
(\mathrm{N}=129)\end{array}$ & $\begin{array}{l}\text { CAC Score }=0 \\
(n=75)\end{array}$ & $\begin{array}{l}\text { CAC Score }>0 \\
(\mathrm{n}=54)\end{array}$ & $P$ Value ${ }^{\mathrm{b}}$ \\
\hline \multicolumn{5}{|l|}{ Psoriasis history } \\
\hline Age at onset, mean (SD), y & $27.8(14.8)$ & $25.2(13.7)$ & $31.4(2.1)$ & .02 \\
\hline Disease duration, median (IQR), y & $11(4-23)$ & $11(4-21)$ & $8.5(5-24)$ & .97 \\
\hline Family history of psoriasis & $56(43.4)$ & $31(41.3)$ & $24(44.4)$ & .72 \\
\hline \multicolumn{5}{|l|}{ Psoriasis type } \\
\hline Plaque psoriasis & $120(93.0)$ & $74(98.7)$ & $46(85.2)$ & .003 \\
\hline Flexural or intertriginous & $40(31.0)$ & $23(30.7)$ & $17(31.5)$ & .92 \\
\hline Seborrheic & $26(20.2)$ & $19(25.3)$ & $7(13.0)$ & .08 \\
\hline Scalp & $109(84.5)$ & $66(88.0)$ & $43(79.6)$ & .20 \\
\hline Palms and/or soles (nonpustular) & $26(20.2)$ & $15(20.0)$ & $11(20.4)$ & .96 \\
\hline Nails & $66(51.2)$ & $35(46.7)$ & $31(57.4)$ & .23 \\
\hline \multicolumn{5}{|l|}{ Psoriasis severity } \\
\hline PASI score, median (IQR) & $1.2(0-3)$ & $0.8(0-2.8)$ & $1.45(0-3.4)$ & .62 \\
\hline BSA score, median (IQR) & $4(2.5-7)$ & $2.5(2-4)$ & $4.5(2.6-8)$ & .36 \\
\hline PGA score & & & & .65 \\
\hline $0 / 1$ & $100(77.5)$ & $60(80.0)$ & $40(74.1)$ & \\
\hline$>1$ & $29(22.5)$ & $15(20.0)$ & $14(25.9)$ & \\
\hline \multicolumn{5}{|l|}{ Treatment history } \\
\hline Duration of treatment, median (IQR), y & $3.0(1.0-5.5)$ & $2.5(1.0-5.0)$ & $3.0(1.0-6.0)$ & .97 \\
\hline Topical therapy only & $53(41.1)$ & $31(41.3)$ & $22(40.7)$ & .95 \\
\hline Phototherapy & $45(34.9)$ & $27(36.0)$ & $26(48.1)$ & .17 \\
\hline \multicolumn{5}{|l|}{ Systemic therapy } \\
\hline Biological & $96(74.4)$ & $56(74.7)$ & $40(74.1)$ & .94 \\
\hline Nonbiological & $42(32.6)$ & $23(30.7)$ & $19(35.2)$ & .59 \\
\hline Psoriatic arthritis & $44(34.1)$ & $28(37.3)$ & 16 (29.6) & .36 \\
\hline
\end{tabular}

Abbreviations: BSA, body surface area; CAC, coronary artery calcium; $\mathrm{IQR}$, interquartile range: PASI, Psoriasis Area and Severity Index; PGA, Physician Global Assessment.

a Data are expressed as number (percentage) of patients unless otherwise indicated.

${ }^{\mathrm{b}} P$ values were derived from a simple unpaired, 2-tailed $t$ test for parametric variables and from the Mann-Whitney test for nonparametric variables. The $\chi^{2}$ test was used for categorical variables. $P<.05$ is considered statistically significant.

Figure. Median Coronary Artery Calcium (CAC) as Assessed by Mean Agatston Scores

A CAC in psoriasis by risk factors

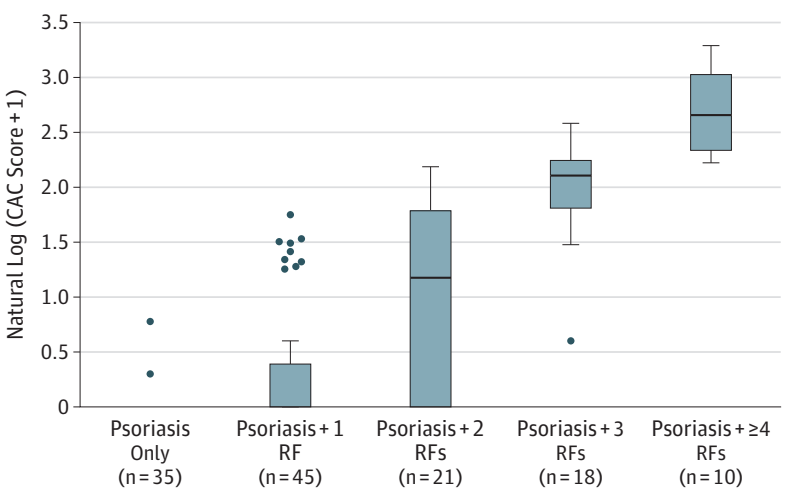

Increases in CAC scores were seen with increasing prevalence of traditional cardiovascular risk factors (RFs) in patients with psoriasis and patients with type 2 diabetes. Traditional cardiovascular RFs are defined as age older than 55 years, hypertension (defined as systolic blood pressure $>140$ mm Hg, diastolic blood pressure $>90 \mathrm{~mm} \mathrm{Hg}$, or normal blood pressure taking antihypertensive medications), hyperlipidemia (defined as high-density lipoprotein cholesterol
B CAC in type 2 diabetes by risk factors

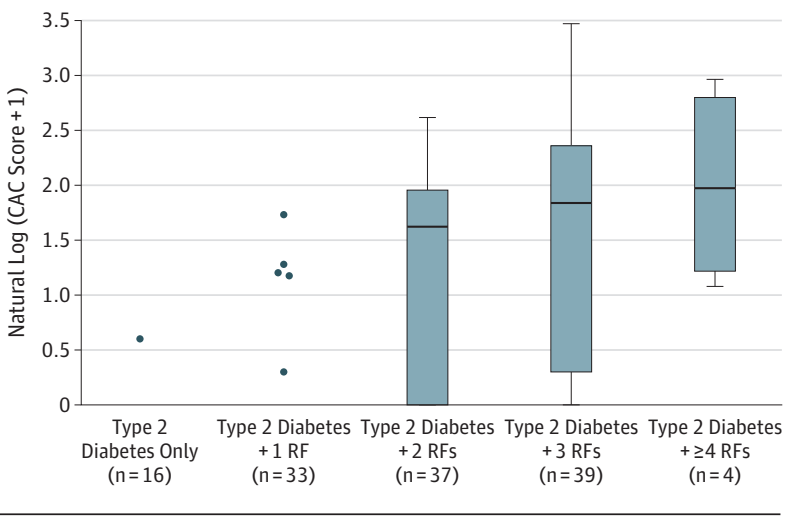

level $<40 \mathrm{mg} / \mathrm{dL}$, low-density lipoprotein cholesterol level $>160 \mathrm{mg} / \mathrm{dL}$ [to convert to millimoles per liter, multiply by 0.0259 ], or triglyceride levels $>200$ $\mathrm{mg} / \mathrm{dL}$ [to convert to millimoles per liter, multiply by 0.0113], or normal lipid profile while taking antihyperlipidemia medications), current smoker, and family history of coronary artery disease. Error bars indicate interquartile ranges. $P$ for trend $<.001$. psoriasis alone. Furthermore, a higher number of risk factors was associated with higher median CAC assessed by mean Agatston score ( $P$ for all between-group comparisons $<.01)$. The overall trend of increasing CAC was strongly significant $(P<.001)$. Similar findings were observed in type 2 diabetes (Figure, B). 


\begin{tabular}{|c|c|c|c|c|c|c|}
\hline \multirow[b]{2}{*}{ Characteristic } & \multicolumn{2}{|c|}{$\begin{array}{l}\text { Psoriasis Cohort } \\
(n=129)\end{array}$} & \multicolumn{2}{|c|}{$\begin{array}{l}\text { Type } 2 \text { Diabetes Cohort } \\
(\mathrm{n}=129)\end{array}$} & \multicolumn{2}{|c|}{$\begin{array}{l}\text { Healthy Controls } \\
(n=129)\end{array}$} \\
\hline & Coefficient & $P$ Value & Coefficient & $P$ Value & Coefficient & $P$ Value \\
\hline Age & 5.43 & $<.001$ & 5.75 & $<.001$ & 6.33 & $<.001$ \\
\hline Male sex & 1.68 & $<.001$ & 1.25 & $<.001$ & 1.05 & .01 \\
\hline Hypertension & 1.73 & $<.001$ & 0.77 & .03 & 0.66 & .09 \\
\hline Hyperlipidemia & 1.73 & .007 & 0.59 & .12 & 0.04 & .96 \\
\hline Metabolic syndrome & 1.02 & .02 & 0.72 & .09 & 0.56 & .22 \\
\hline Current smoking & 0.83 & .22 & 1.02 & .04 & 0.01 & .99 \\
\hline Regular exercise & -0.35 & .40 & 0.73 & .04 & -0.45 & .25 \\
\hline \multicolumn{7}{|l|}{ Clinical parameters } \\
\hline $\mathrm{BMI}^{\mathrm{b}}$ & 3.50 & .001 & 0.34 & .72 & 0.97 & .43 \\
\hline Waist circumference & 4.74 & $<.001$ & 1.96 & .11 & 2.70 & .07 \\
\hline \multicolumn{7}{|l|}{$\mathrm{BP}$} \\
\hline Systolic & 4.72 & .002 & -0.90 & .57 & 2.22 & .15 \\
\hline Diastolic & 3.659 & .01 & -0.59 & .68 & 0.61 & .69 \\
\hline FRS 10 -y risk & 1.78 & $<.001$ & 1.00 & $<.001$ & 1.20 & $<.001$ \\
\hline \multicolumn{7}{|l|}{ Lipids and glucose profile } \\
\hline Total cholesterol & -1.81 & .09 & -0.91 & .30 & 0.46 & .68 \\
\hline LDL-C & 0.06 & .94 & -1.17 & .07 & 0.42 & .60 \\
\hline HDL-C & -2.52 & $<.001$ & -1.53 & .05 & -1.00 & .16 \\
\hline Triglycerides & 0.68 & .12 & 0.93 & .02 & 0.73 & .08 \\
\hline VLDL-C & 3.46 & .08 & 0.70 & .03 & 0.55 & .10 \\
\hline Glucose & 4.96 & .01 & -0.03 & .96 & 2.05 & .11 \\
\hline
\end{tabular}

Abbreviations: BMI, body mass index; $\mathrm{BP}$, blood pressure; CAC, coronary artery calcium; FRS, Framingham Risk Score; HDL-C, high-density lipoprotein cholesterol; LDL-C, low-density lipoprotein cholesterol; VLDL-C, very low-density lipoprotein cholesterol.

a $P<.05$ is considered statistically significant. All characteristics in the table were log transformed and used in the Tobit regression models. ${ }^{b}$ Calculated as weight in kilograms divided by height in meters squared.
Association of CAC in Psoriasis With Cardiovascular and Cardiometabolic Risk Factors

In unadjusted Tobit regression analysis of CAC compared with variables of interest, all 3 groups had significant correlations between CAC and age, male sex, and FRS 10-year risk, as would be expected (Table 3 ). The CAC in the psoriasis cohort revealed stronger correlations with cardiovascular and cardiometabolic risk factors, including hypertension (Tobit regression ratio [TRR], 1.73, 95\% CI, 0.96-2.51; $P<.001$ ), metabolic syndrome (TRR, 1.02; 95\% CI, 0.20-1.83; $P=.02$ ), waist circumference (TRR, 4.74; 95\% CI, 2.15-6.93; $P<.001$ ), systolic (TRR, 4.72, 95\% CI, 1.76-7.69; $P$ = .002) and diastolic (TRR, 3.66; 95\% CI, 0.88-6.42; $P=.01$ ) blood pressures, FRS 10-year risk (TRR, 1.78; 95\% CI, 1.00-2.55; $P<.001$ ), HDL-C level (TRR, $-2.52 ; 95 \%$ CI, -3.81 to $-1.22 ; P<.001)$, and blood glucose level (TRR, 4.96; 95\% CI, 1.14-8.78; $P=.01$ ) when compared with the age- and sex- matched type 2 diabetes group. In the type 2 diabetes cohort, CAC scores more strongly correlated with current smoking (TRR, 1.02; 95\% CI, 0.01-2.04; $P=.04$ ), regular exercise (TRR, 0.73; 95\% CI, 0.01-1.45; $P=.04$ ), triglycerides level (TRR, 0.93; 95\% CI, 0.18-1.67; $P=.02$ ), and very lowdensity lipoprotein cholesterol level (TRR, 0.70; 95\% CI, 0.05$1.34 ; P=.03)$. Spearman correlation analysis also revealed similar associations as univariate regression analysis (eTable 1 in the Supplement).

\section{Association of Psoriasis With CAC in Models \\ Adjusted for Cardiometabolic Risk Factors}

Hierarchical Tobit regression analysis (Table 4) was performed to define the association between the presence of dis- ease (psoriasis or type 2 diabetes) and CAC after adjusting for groups of confounding variables. In unadjusted analysis, type 2 diabetes (TRR, 1.12; 95\% CI, 0.57-1.67; $P<.001$ ) had a stronger association with CAC than psoriasis (TRR, 0.73; 95\% CI, 0.18-1.29; $P=.01$ ). However, in the final model, which was fully adjusted for age, sex, cardiovascular risk factors (LDL-C, HDL-C, triglycerides, and fasting blood glucose levels, systolic blood pressure, and tobacco use), cardiometabolic risk factors (BMI and metabolic syndrome), and medication use (aspirin, angiotensin-converting enzyme inhibitors, and statins), psoriasis was associated with CAC (TRR, 0.89; 95\% CI, 0.35-1.44; $P=.001$ ) similar to the association in type 2 diabetes (TRR, 0.79; 95\% CI, 0.02-1.55; $P=.04$ ).

Logistic multivariable regression models (Table 5) were constructed using the same models as in the Tobit regression analysis, with CAC score greater than $\mathrm{O}$ as the outcome. Psoriasis was associated with the presence of any CAC after adjusting for age, sex, and cardiovascular risk factors, metabolic risk factors, and medication use (OR, 2.35; 95\% CI, 1.124.94), which attenuated when BMI was added to the model (OR, 2.18; 95\% CI, 0.75-6.35) in type 2 diabetes. Furthermore, when we added use of systemic or biological therapy to the models, the TRR and OR increased; however, these analyses were exploratory.

\section{Discussion}

We report 4 salient findings from our comparison of patients with psoriasis, patients with type 2 diabetes, and healthy con- 


\begin{tabular}{|c|c|c|c|c|c|c|c|c|}
\hline \multirow[b]{2}{*}{ Model $^{\mathrm{a}}$} & \multicolumn{2}{|c|}{ Psoriasis } & \multirow[b]{2}{*}{$\chi^{2}$ Test } & \multirow[b]{2}{*}{$P$ Value } & \multicolumn{2}{|c|}{ Type 2 Diabetes } & \multirow[b]{2}{*}{$x^{2}$ Test } & \multirow[b]{2}{*}{$P$ Value $^{\mathrm{b}}$} \\
\hline & Ratio & $P$ Value $^{\mathrm{b}}$ & & & Ratio & $P$ Value $^{\mathrm{b}}$ & & \\
\hline Unadjusted & 0.73 & .01 & NA & NA & 1.12 & $<.001$ & NA & NA \\
\hline Model 1 & 0.73 & .002 & 9.15 & .003 & 1.10 & $<.001$ & 22.37 & $<.001$ \\
\hline Model 2 & 0.85 & .002 & 3.48 & .06 & 0.90 & .01 & 4.88 & .02 \\
\hline Model 3 & 0.93 & .001 & 4.05 & .04 & 0.85 & .02 & 3.34 & .06 \\
\hline Model 4 & 0.89 & .001 & 4.48 & .03 & 0.79 & .04 & 1.54 & .21 \\
\hline Model 5 & 1.30 & .01 & 4.50 & .03 & NA & NA & NA & NA \\
\hline \multicolumn{5}{|c|}{$\begin{array}{l}\text { Abbreviation: NA, not applicable. } \\
\text { a Model } 1 \text { was adjusted for age and sex; model } 2 \text { was adjusted for age, sex, and } \\
\text { cardiovascular risk factors (low-density lipoprotein cholesterol level, } \\
\text { high-density lipoprotein cholesterol level, triglycerides level, systolic blood } \\
\text { pressure, fasting blood glucose level, and current tobacco use); model } 3 \text { was } \\
\text { adjusted for age, sex, and cardiovascular and cardiometabolic risk factors } \\
\text { (body mass index and metabolic syndrome); model } 4 \text { was adjusted for }\end{array}$} & \multicolumn{4}{|c|}{$\begin{array}{l}\text { demographics, cardiovascular and cardiometabolic risk factors, and } \\
\text { medication use (aspirin, angiotensin-converting enzyme inhibitors, and } \\
\text { statins); and model } 5 \text { was adjusted for demographics, cardiovascular and } \\
\text { cardiometabolic risk factors, medication use, and psoriasis and rheumatologic } \\
\text { treatment (methotrexate, any systemic, and any biological treatment). All } \\
\text { variables were log transformed before use in the Tobit regression models. }\end{array}$} \\
\hline
\end{tabular}

Table 5. Logistic Regression Analysis of Coronary Artery Calcium Score Greater Than 0 With Psoriasis and Type 2 Diabetes

\begin{tabular}{lll}
\hline \multirow{2}{*}{ Model $^{\mathrm{a}}$} & \multicolumn{2}{l}{ Odds Ratio $(95 \% \mathrm{Cl})$} \\
\cline { 2 - 3 } & Psoriasis Cohort & Type 2 Diabetes Cohort \\
\hline Unadjusted & $1.60(0.9-2.7)$ & $2.26(1.4-3.8)^{\mathrm{b}}$ \\
\hline Model 1 & $1.82(1.0-3.3)^{\mathrm{b}}$ & $2.89(1.6-5.2)^{\mathrm{b}}$ \\
\hline Model 2 & $2.17(1.1-4.4)^{\mathrm{b}}$ & $2.41(0.9-6.3)$ \\
\hline Model 3 & $2.37(1.1-5.0)^{\mathrm{b}}$ & $2.23(0.8-6.0)$ \\
\hline Model 4 & $2.35(1.12-4.94)^{\mathrm{b}}$ & $2.18(0.8-6.4)$ \\
\hline Model 5 & $3.99(1.0-16.1)^{\mathrm{b}}$ & $\mathrm{NA}$ \\
\hline
\end{tabular}

Abbreviation: NA, not applicable.

${ }^{\text {a }}$ Model 1 was adjusted for age and sex; model 2 was adjusted for age, sex, and cardiovascular risk factors (low-density lipoprotein cholesterol level, high-density lipoprotein cholesterol level, triglycerides level, systolic blood pressure, fasting blood glucose level, and current tobacco use); model 3 was adjusted for age, sex, and cardiovascular and cardiometabolic risk factors (body mass index and metabolic syndrome); model 4 was adjusted for demographics, cardiovascular and cardiometabolic risk factors, and medication use (aspirin, angiotensin-converting enzyme inhibitors, and statins); and model 5 was adjusted for demographics, cardiovascular and cardiometabolic risk factors, medication use, and psoriasis and rheumatologic treatment (methotrexate, any systemic, and any biological treatment). All variables were log transformed before use in the Tobit regression models. ${ }^{\mathrm{b}} P \leq .05$ is considered statistically significant.

trols: (1) cardiovascular risk factor profiles were similar in patients with psoriasis and type 2 diabetes when compared with age- and sex-matched controls; (2) the prevalence of moderate to severe coronary calcification (CAC mean total Agatston score $>100$ and $>400$ ) was similar between patients with psoriasis and type 2 diabetes and approximately 5 times greater than controls; (3) coronary calcium in patients with moderate to severe psoriasis demonstrated a similar association with known cardiovascular and cardiometabolic risk factors when compared with type 2 diabetes; and (4) the presence of CAC in patients with psoriasis persisted independent of BMI, which was not observed in patients with type 2 diabetes.

Psoriasis and type 2 diabetes share common pathogenetic mechanisms with the important potential for inflammatory atherogenesis. Both are chronic inflammatory disorders that result from a complex interplay between genetic and environmental influences. They both demonstrate a predominantly proinflammatory milieu composed of tumor necrosis factor, interferon $\gamma$, interleukin (IL) 6, and IL-17 caused by imbalances in proinflammatory (helper $\mathrm{T}$ cell $\left[\mathrm{T}_{\mathrm{H}} 1\right]$ and $\mathrm{T}_{\mathrm{H}} 17$ ) and anti-inflammatory (T_regulatory and $\mathrm{T}_{\mathrm{H}} 2$ ) $\mathrm{T}$-cell subsets and increased expression of proinflammatory signature genes, resulting in a chronic inflammatory state. ${ }^{32,33}$ This inflammation contributes to endothelial dysfunction and monocyte or macrophage recruitment, seminal events in atherogenesis. ${ }^{34}$ Elevated markers of oxidative stress and platelet-derived microparticles have been reported in the serum of patients with psoriasis ${ }^{35}$ and type 2 diabetes, ${ }^{36}$ which can lead to increased endothelial activation. ${ }^{34}$ Several epidemiologic studies ${ }^{37-39}$ have described the high incidence of cardiovascular risk factors, such as smoking, hypertension, dyslipidemia, and central obesity, in both of these groups. Obesity and the associated metabolic syndrome are especially important because adipose tissue is a major source of tumor necrosis factor, IL-1, IL-6, IL-8, and monocyte chemoattractant protein 1. Adipose tissues also secrete adipokines, including leptin and resistin, which can independently lead to insulin resistance and increased risk of atherogenesis. ${ }^{34}$

An increased incidence of thrombo-occlusive disease was first noted in patients with psoriasis in the late $1970 \mathrm{~s} .{ }^{40}$ Most of the data, which have driven the discussion linking psoriasis with cardiovascular disease, prognostic indicators, and patient management strategies, have been accrued during the past decade. ${ }^{41}$ Multiple oral systemic and biological treatment strategies have produced decreased vascular inflammation with appropriate patient management in psoriasis and other immune-mediated inflammatory diseases. In recent years, various noninvasive imaging and functional markers have been studied to better understand the risk for atherosclerosis in psoriasis. ${ }^{14,42,43}$ Multidetector computed tomography-derived CAC has long been recognized as a reliable surrogate marker of subclinical atherosclerosis and has immense prognostic value, especially in asymptomatic adults at intermediate cardiovascular risk. ${ }^{44}$ Coronary artery calcium has high sensitivity, albeit only moderate specificity, for obstructive CAD. In addition, calcification is a measure of stabilized 
plaque but may not always account for the noncalcified plaque burden (ie, the type that is vulnerable for rupture). Nonetheless, studies have found that CAC can reliably predict future adverse cardiovascular events, correlates well with cardiac computed tomography angiography findings of significant angiographic stenosis, ${ }^{45}$ is cost-effective, ${ }^{46}$ and provides an additive benefit to existing cardiovascular risk assessment tools. ${ }^{47}$

The beneficial role of CAC in cardiovascular risk assessment of type 2 diabetes has been well established. ${ }^{25}$ However, CAC assessment in psoriasis has only been performed in a small number of case-control studies ${ }^{18-20,48,49}$ (eTable 2 in the Supplement). Similar to our results, most of these studies report an increased CAC burden in patients with psoriasis when compared with controls. Although a previous study ${ }^{50}$ found a lower risk for cardiovascular events in patients with psoriasis treated with anti-tumor necrosis factor therapy, no study to date has investigated the longitudinal effect of systemic therapy on CAC. In the present study, we found that adjustment for any systemic and biological therapy in multivariable model increased the regression ratio and OR by approximately $50 \%$, thereby suggesting that these treatments may in fact have an association with lower CAC in patients with psoriasis. Ongoing studies ${ }^{51-53}$ in psoriasis are investigating the effect of intensive skin disease treatment on cardiovascular disease (clinicaltrials.gov, NCT01553058, NCT02187172, and NCT01866592). These studies will elucidate the effect of biological treatment on subclinical vascular diseases.

In a landmark study, Gelfand et $\mathrm{al}^{5}$ found a significantly high incidence of myocardial infarction in patients with psoriasis from a UK-based registry of more than 500000 patients. To our knowledge, our study is the first to investigate the prevalence of CAC in a large cohort of patients with psoriasis when compared with a matched type 2 diabetes cohort, a well-established coronary disease risk equivalent. We were also able to demonstrate a strong association between subclinical atherosclerosis and prevalence of cardiovascular and cardiometabolic risk factors in psoriasis. Our findings are certainly intriguing given the fact that our patients were relatively young, with most having their moderate to severe cutaneous disease under good therapeutic control. However, the relative presence of moderate to severe psoriasis revealed greater strength in CAC assessment than type 2 diabetes, in- dependent of the effect of known cardiovascular and cardiometabolic risk factors. The attenuation of the association between type 2 diabetes and CAC when adjusted for BMI was not observed in psoriasis, suggesting that CAC may be mediated, in part, by adiposity in type 2 diabetes.

We acknowledge certain limitations relating to our study design. The cross-sectional study design and lack of biological data limit our ability to establish a cause-effect relationship between psoriasis and atherosclerosis. Variations among different ethnic groups could not be established given that most of our study patients were white, which is the major demographic group affected with psoriasis in North America and Europe. Of note, almost all patients with psoriasis controlled their disease well with systemic or biological therapy for approximately 3 years before recruitment. This level of disease control likely underestimates the burden of subclinical atherosclerosis in our cohort. In addition, high-sensitivity C-reactive protein values were not obtained for our cohort of patients with psoriasis. As such, we were unable to adjust for this variable as a crude marker of inflammation in our models. Furthermore, a previous study by Staniak et $\mathrm{al}^{19}$ found a doseresponse relationship between psoriatic skin severity and severe coronary calcium disease (CAC score $>400$ ). However, our study failed to find this relationship, likely because of the wellcontrolled nature of cutaneous disease plus the inclusion of previously treated patients in our study when compared with the cohort of patients with psoriasis.

\section{Conclusions}

Psoriasis increases CAC scores to the extent of what is observed in type 2 diabetes, independent of the effect of cardiovascular and cardiometabolic risk factors. Psoriasis and type 2 diabetes share similar cardiovascular risk profiles, which may predispose patients to developing coronary atherosclerosis at a relatively young age. These findings warrant early cardiovascular risk assessment and aggressive risk factor modification in those with moderate to severe psoriasis. In addition, CAC assessment may be considered in patients with psoriasis who have 2 or more traditional cardiovascular risk factors given the high prevalence of CAC observed in this study.

\section{ARTICLE INFORMATION}

Accepted for Publication: June 21, 2016.

Published Online: August 24, 2016. doi:10.1001/jamadermatol.2016.2907.

Author Affiliations: Division of Dermatology, Baylor University Medical Center, Dallas, Texas (Mansouri, Kivelevitch, Ryan, Menter); Department of Dermatology, Baylor Scott and White Health, Temple, Texas (Mansouri); Baylor Institute for Immunology Research, Dallas, Texas (Kivelevitch); University of Arizona College of Medicine at South Campus, Tucson (Natarajan); National Heart, Lung and Blood Institute, National Institutes of Health, Bethesda, Maryland (Natarajan, Joshi, Mehta); Texas A\&M Health Science Center College of Medicine, Temple (Benjegerdes); Division of Cardiology, Baylor University Medical Center,
Dallas, Texas (Schussler); Baylor Jack and Jane Hamilton Heart and Vascular Hospital, Dallas, Texas (Schussler); Texas A\&M Health Science Center College of Medicine, Dallas (Schussler); Perelman School of Medicine, University of Pennsylvania, Philadelphia (Rader, Reilly); Division of Cardiology, Department of Medicine, Columbia University, New York, New York (Reilly); Irving Institute for Clinical and Translational Research, Columbia University, New York, New York (Reilly).

Author Contributions: Dr Mehta had full access to all of the data in the study and takes responsibility for the integrity of the data and the accuracy of the data analysis.

Study concept and design: Mansouri, Ryan Schussler, Rader, Reilly, Menter, Mehta. Acquisition, analysis, or interpretation of data: Mansouri, Kivelevitch, Natarajan, Joshi, Ryan,
Benjegerdes, Schussler, Reilly, Menter, Mehta. Drafting of the manuscript: Mansouri, Natarajan, Ryan, Benjegerdes, Schussler, Menter, Mehta. Critical revision of the manuscript for important intellectual content: All authors. Statistical analysis: Natarajan, Joshi, Benjegerdes, Mehta.

Obtaining funding: Mansouri, Kivelevitch, Ryan, Reilly, Menter, Mehta.

Administrative, technical, or material support: Mansouri, Kivelevitch, Joshi, Benjegerdes, Reilly, Mehta.

Study supervision: Mansouri, Kivelevitch, Ryan, Schussler, Menter.

Conflict of Interest Disclosures: Dr Mansouri reported serving on an advisory board for Celgene, receiving an honorarium. Dr Ryan reported serving 
as an adviser and/or speaker for Abbvie, Aqua, Lilly, Medimetriks, Novartis, Regeneron-Sanofi, and UCB, receiving honoraria for all. Dr Menter reported serving as a consultant or investigator for Abbott Laboratories, receiving honoraria; serving on advisory boards or as a speaker for Abbott Laboratories, AbbVie, Amgen, Astellas Pharma US Inc, Centocor Ortho Biotech Inc, Galderma Laboratories LP, Pfizer Inc, and Warner Chilcott, receiving honoraria or fees; and reported pending grants from Amgen, Celgene Corporation, Centocor Ortho Biotech Inc, and Pfizer Inc. Dr Mehta reported being is a full-time US government employee. No other disclosures were reported.

Funding/Support: This study was supported in part by the Baylor Heart and Vascular Institute Cardiovascular Research Review Committee and The Jack and Jane Hamilton Cardiovascular Research Fund and by grant HL006193-002 from the Intramural Research Program at the National Institutes of Health (Dr Mehta)

Role of the Funder/Sponsor: The funding sources had no role in the design and conduct of the study; collection, management, analysis, and interpretation of the data; preparation, review, or approval of the manuscript; and the decision to submit the manuscript for publication.

Additional Contributions: Cristina Martinez, MD, significantly contributed to research patient coordination. Connie Tran, MD, and Lindsey Richards, MD, performed data entry and database management. None were compensated for their contributions.

\section{REFERENCES}

1. Parisi R, Symmons DP, Griffiths CE, Ashcroft DM; Identification and Management of Psoriasis and Associated ComorbidiTy (IMPACT) project team. Global epidemiology of psoriasis: a systematic review of incidence and prevalence. J Invest Dermatol. 2013;133(2):377-385.

2. Menter A, Gottlieb A, Feldman SR, et al. Guidelines of care for the management of psoriasis and psoriatic arthritis, section 1: overview of psoriasis and guidelines of care for the treatment of psoriasis with biologics. J Am Acad Dermatol. 2008 58(5):826-850

3. Armstrong AW, Harskamp CT, Armstrong EJ. Psoriasis and metabolic syndrome: a systematic review and meta-analysis of observational studies. J Am Acad Dermatol. 2013;68(4):654-662.

4. Armstrong EJ, Harskamp CT, Armstrong AW. Psoriasis and major adverse cardiovascular events: a systematic review and meta-analysis of observational studies. J Am Heart Assoc. 2013;2(2): e000062.

5. Gelfand JM, Neimann AL, Shin DB, Wang X, Margolis DJ, Troxel AB. Risk of myocardial infarction in patients with psoriasis. JAMA. 2006;296(14): 1735-1741.

6. Gelfand JM, Dommasch ED, Shin DB, et al. The risk of stroke in patients with psoriasis. J Invest Dermatol. 2009;129(10):2411-2418.

7. Prodanovich S, Kirsner RS, Kravetz JD, Ma F, Martinez L, Federman DG. Association of psoriasis with coronary artery, cerebrovascular, and peripheral vascular diseases and mortality. Arch Dermatol. 2009;145(6):700-703.

8. Mehta NN, Azfar RS, Shin DB, Neimann AL, Troxel AB, Gelfand JM. Patients with severe psoriasis are at increased risk of cardiovascular mortality: cohort study using the General Practice Research Database. Eur Heart J. 2010;31(8):1000 1006.

9. Abuabara K, Azfar RS, Shin DB, Neimann AL, Troxel AB, Gelfand JM. Cause-specific mortality in patients with severe psoriasis: a population-based cohort study in the U.K. Br J Dermatol. 2010;163(3): 586-592.

10. Ogdie A, Yu Y, Haynes K, et al. Risk of major cardiovascular events in patients with psoriatic arthritis, psoriasis and rheumatoid arthritis: a population-based cohort study. Ann Rheum Dis. 2015;74(2):326-332

11. Mehta NN, Yu Y, Pinnelas R, et al. Attributable risk estimate of severe psoriasis on major cardiovascular events. Am J Med. 2011;124(8):775, e771-e776.

12. Langan SM, Seminara NM, Shin DB, et al. Prevalence of metabolic syndrome in patients with psoriasis: a population-based study in the United Kingdom. J Invest Dermatol. 2012;132(3, pt 1):556 562.

13. Patel RV, Shelling ML, Prodanovich S, Federman DG, Kirsner RS. Psoriasis and vascular disease-risk factors and outcomes: a systematic review of the literature. J Gen Intern Med. 2011;26(9):1036-1049.

14. Dave J, Ahlman MA, Lockshin BN, Bluemke DA, Mehta NN. Vascular inflammation in psoriasis localizes to the arterial wall using a novel imaging technique. J Am Acad Dermatol. 2014;70(6):11371138.

15. Mehta NN, Yu Y, Saboury B, et al. Systemic and vascular inflammation in patients with moderate to severe psoriasis as measured by [18F]fluorodeoxyglucose positron emission tomography-computed tomography (FDG-PET/CT): a pilot study. Arch Dermatol. 2011;147(9):1031-1039. 16. Detrano R, Guerci AD, Carr JJ, et al. Coronary calcium as a predictor of coronary events in four racial or ethnic groups. N Engl J Med. 2008;358(13): 1336-1345

17. Okwuosa TM, Greenland $\mathrm{P}$, Ning $\mathrm{H}$, et al. Distribution of coronary artery calcium scores by Framingham 10-year risk strata in the MESA (Multi-Ethnic Study of Atherosclerosis) potential implications for coronary risk assessment. J Am Coll Cardiol. 2011;57(18):1838-1845.

18. Ludwig RJ, Herzog C, Rostock A, et al. Psoriasis: a possible risk factor for development of coronary artery calcification. Br J Dermatol. 2007;156(2):271276.

19. Staniak HL, Bittencourt MS, de Souza Santos I, et al. Association between psoriasis and coronary calcium score. Atherosclerosis. 2014;237(2):847-852.

20. Yiu KH, Yeung $\mathrm{CK}$, Zhao $\mathrm{CT}$, et al. Prevalence and extent of subclinical atherosclerosis in patients with psoriasis. J Intern Med. 2013;273(3):273-282.

21. Mehta NN, Li R, Krishnamoorthy P, et al. Abnormal lipoprotein particles and cholesterol efflux capacity in patients with psoriasis. Atherosclerosis. 2012;224(1):218-221.

22. Li RC, Krishnamoorthy P, DerOhannessian S et al. Psoriasis is associated with decreased plasma adiponectin levels independently of cardiometabolic risk factors. Clin Exp Dermatol. 2014;39(1):19-24.

23. Anand DV, Lim E, Hopkins D, et al. Risk stratification in uncomplicated type 2 diabetes: prospective evaluation of the combined use of coronary artery calcium imaging and selective myocardial perfusion scintigraphy. Eur Heart $J$. 2006;27(6):713-721.

24. Becker A, Leber AW, Becker C, et al. Predictive value of coronary calcifications for future cardiac events in asymptomatic patients with diabetes mellitus: a prospective study in 716 patients over 8 years. BMC Cardiovasc Disord. 2008;8:27.

25. Elkeles RS, Godsland IF, Feher MD, et al; PREDICT Study Group. Coronary calcium measurement improves prediction of cardiovascular events in asymptomatic patients with type 2 diabetes: the PREDICT study. Eur Heart J. 2008:29(18):2244-2251.

26. Greenland P, Alpert JS, Beller GA, et al; American College of Cardiology Foundation/ American Heart Asscoiation Tasl Force on Practice Guidelines. 2010 ACCF/AHA guideline for assessment of cardiovascular risk in asymptomatic adults: executive summary: a report of the American College of Cardiology Foundation/ American Heart Association Task Force on Practice Guidelines. Circulation. 2010;122(25):2748-2764.

27. Reilly MP, lqbal N, Schutta M, et al. Plasma leptin levels are associated with coronary atherosclerosis in type 2 diabetes. J Clin Endocrinol Metab. 2004;89(8):3872-3878.

28. Grundy SM, Cleeman JI, Daniels SR, et al; American Heart Association; National Heart, Lung, and Blood Institute. Diagnosis and management of the metabolic syndrome: an American Heart Association/National Heart, Lung, and Blood Institute Scientific Statement. Circulation. 2005;112 (17):2735-2752.

29. Wilson PW, D'Agostino RB, Levy D, Belanger AM, Silbershatz $H$, Kannel WB. Prediction of coronary heart disease using risk factor categories Circulation. 1998;97(18):1837-1847.

30. Agatston AS, Janowitz WR, Hildner FJ, Zusmer NR, Viamonte M Jr, Detrano R. Quantification of coronary artery calcium using ultrafast computed tomography. J Am Coll Cardiol. 1990;15(4):827-832. 31. Tobin J. Estimation of relationships for limited dependent variables. Econometrica. 1958;26(1):2436.

32. Dowlatshahi EA, van der Voort EA, Arends LR, Nijsten T. Markers of systemic inflammation in psoriasis: a systematic review and meta-analysis. $\mathrm{Br}$ J Dermatol. 2013;169(2):266-282.

33. Jagannathan-Bogdan M, McDonnell ME, Shin $\mathrm{H}$, et al. Elevated proinflammatory cytokine production by a skewed T cell compartment requires monocytes and promotes inflammation in type 2 diabetes. J Immunol. 2011;186(2):1162-1172.

34. Galkina E, Ley K. Immune and inflammatory mechanisms of atherosclerosis $\left({ }^{*}\right)$. Annu Rev Immunol. 2009;27:165-197.

35. Takeshita J, Mohler ER, Krishnamoorthy P, et al. Endothelial cell-, platelet-, and monocyte/ macrophage-derived microparticles are elevated in psoriasis beyond cardiometabolic risk factors. J Am Heart Assoc. 2014;3(1):e000507.

36. Nomura S, Suzuki M, Katsura K, et al. Platelet-derived microparticles may influence the development of atherosclerosis in diabetes mellitus. Atherosclerosis. 1995;116(2):235-240. 37. Armstrong AW, Harskamp CT, Armstrong EJ. The association between psoriasis and obesity: a systematic review and meta-analysis of observational studies. Nutr Diabetes. 2012;2:e54. 
38. Neimann AL, Shin DB, Wang X, Margolis DJ, Troxel AB, Gelfand JM. Prevalence of cardiovascular risk factors in patients with psoriasis. J Am Acad Dermatol. 2006;55(5):829-835.

39. Sarwar N, Gao P, Seshasai SR, et al; Emerging Risk Factors Collaboration. Diabetes mellitus, fasting blood glucose concentration, and risk of vascular disease: a collaborative meta-analysis of 102 prospective studies [published correction appears in in Lancet. 2010;376(9745):958]. Lancet. 2010;375(9733):2215-2222.

40. McDonald CJ, Calabresi P. Psoriasis and occlusive vascular disease. Br J Dermatol. 1978;99 (5):469-475.

41. Bissonnette R, Tardif JC, Harel F, Pressacco J, Bolduc C, Guertin MC. Effects of the tumor necrosis factor-a antagonist adalimumab on arterial inflammation assessed by positron emission tomography in patients with psoriasis: results of a randomized controlled trial. Circ Cardiovasc Imaging. 2013;6(1):83-90.

42. Salahuddin T, Natarajan B, Playford MP, et al. Cholesterol efflux capacity in humans with psoriasis is inversely related to non-calcified burden of coronary atherosclerosis. Eur Heart J. 2015;36(39): 2662-2665.
43. Robati RM, Partovi-Kia M, Haghighatkhah HR, Younespour S, Abdollahimajd F. Increased serum leptin and resistin levels and increased carotid intima-media wall thickness in patients with psoriasis: is psoriasis associated with atherosclerosis? J Am Acad Dermatol. 2014;71(4): 642-648.

44. Greenland P, Alpert JS, Beller GA, et al; American College of Cardiology Foundation; American Heart Association. 2010 ACCF/AHA guideline for assessment of cardiovascular risk in asymptomatic adults: a report of the American College of Cardiology Foundation/American Heart Association Task Force on Practice Guidelines. J Am Coll Cardiol. 2010;56(25):e50-e103.

45. Baumgart D, Schmermund A, Goerge G, et al. Comparison of electron beam computed tomography with intracoronary ultrasound and coronary angiography for detection of coronary atherosclerosis. J Am Coll Cardiol. 1997;30(1):57-64.

46. Zeb I, Abbas N, Nasir K, Budoff MJ. Coronary computed tomography as a cost-effective test strategy for coronary artery disease assessment: a systematic review. Atherosclerosis. 2014;234(2): 426-435.

47. Polonsky TS, McClelland RL, Jorgensen NW, et al. Coronary artery calcium score and risk classification for coronary heart disease prediction. JAMA. 2010;303(16):1610-1616.

48. Torres T, Bettencourt N, Mendonça D, et al. Epicardial adipose tissue and coronary artery calcification in psoriasis patients. J Eur Acad Dermatol Venereol. 2015;29(2):270-277.

49. Hjuler KF, Böttcher M, Vestergaard C, et al. Increased prevalence of coronary artery disease in severe psoriasis and severe atopic dermatitis. Am J Med. 2015;128(12):1325-1334.

50. Wu JJ, Poon KY. Association of gender, tumor necrosis factor inhibitor therapy, and myocardial infarction risk in patients with psoriasis. $J$ Am Acad Dermatol. 2013;69(4):650-651.

51. clinicaltrials.gov. Vascular Inflammation in Psoriasis Trial (The VIP Trial) (VIP). NCT01553058. https://clinicaltrials.gov/ct2/show/NCT01553058. Accessed June 1, 2016.

52. clinicaltrials.gov. Vascular Inflammation in Psoriasis-Ustekinumab (VIP-U) (VIP-U). NCTO2187172. https://clinicaltrials.gov/ct2/show /NCTO2187172. Accessed June 1, 2016.

53. clinicaltrials.gov. Vascular Inflammation in Psoriasis - Extension Study (VIP-E). NCT01866592. https://clinicaltrials.gov/ct2/show/NCT01866592. Accessed June 1, 2016.

\section{NOTABLE NOTES}

\section{The Legacy of Maurice Raynaud}

\section{Arpan V. Prabhu, BS; Chester V. Oddis, MD}

Born in 1834 as the son of a university professor, Maurice Raynaud trained at the University of Paris and became a French physician in $1862 .{ }^{1} \mathrm{Al}-$ though he was affiliated with various hospitals and clinics, for political reasons Raynaud was never able to secure a senior faculty position at any hospitals in Paris. Nevertheless, he achieved scientific and medical fame as a medical student with his doctoral thesis titled, "Local Asphyxia and Symmetrical Gangrene of the Extremities." Raynaud's thesis described 25 patients, 20 of whom were female, who had a series of color changes in their hands and feet when exposed to the cold or when the patients were under stress. ${ }^{2}$ This is known today as Raynaud phenomenon, and it is associated with a wide variety of medical conditions spanning across multiple medical disciplines. ${ }^{3}$

Raynaud described the order of changes in the extremities from blanching to cyanosis to erythema. ${ }^{3}$ Subsequent investigation has shown that Raynaud phenomenon is mostly triggered by episodic, symmetric vasospasm of the digital arteries leading to the appearance of flat nails. ${ }^{2}$ At the time of his report, Raynaud had not yet recognized that these symptoms could be warning signs of more severe systemic disease, prompting new designations of primary and secondary Raynaud syndromes. ${ }^{1}$ When Raynaud discovered the syndrome, treatment mainly consisted of counseling to avoid cold weather, stressful conditions, and the consumption of anything that would constrict blood vessels. Today, in addition to common sense measures in the cold weather, there are many pharmacologic interventions as well as surgical procedures available as treatment options. $^{3}$
Raynaud's contributions did not end with his report of this syndrome. A year after receiving his doctoral degree, Raynaud submitted a postdoctoral thesis of 2 notable publications on the history of medicine, the "Asclepiades of Bithynia, doctor and philosopher" and "Medicine in Molière's time."1 He was also a prolific writer, and his book Sur la salive d'un enfant mort de la rage was published after a research collaboration with Louis Pasteur and Odilon Marc Lannelongue. In addition, Raynaud was admired and respected as a superb teacher and lecturer throughout the universities and hospitals in Paris. In 1879, he was elected to the Academy of Medicine. ${ }^{1}$

Despite his numerous achievements, Raynaud was unable to achieve his goal of becoming a chair of medical history in Paris. Cardiac disease plagued Raynaud for several years, and he died in 1881 from an acute myocardial infarction.

Author Affiliations: University of Pittsburgh School of Medicine, Pittsburgh, Pennsylvania (Prabhu); Division of Rheumatology and Clinical Immunology, University of Pittsburgh School of Medicine, Pittsburgh, Pennsylvania (Oddis).

Corresponding Author: Arpan V. Prabhu, BS, University of Pittsburgh School of Medicine, 518 Scaife Hall, 3550 Terrace St, Pittsburgh, PA 15213 (prabhuav2 @upmc.edu).

1. Kaiser H. Maurice Raynaud (1834-1881) und das nach ihm benannte Syndrom. Z Rheumatol. 2011;70(7):620-624.

2. Davis EP. The American Journal of the Medical Sciences. Vol 108. Philadelphia, PA: J. B. Lippincott; 1894.

3. Bakst R, Merola JF, Franks AG Jr, Sanchez M. Raynaud's phenomenon: pathogenesis and management. J Am Acad Dermatol. 2008;59(4):633-653. 